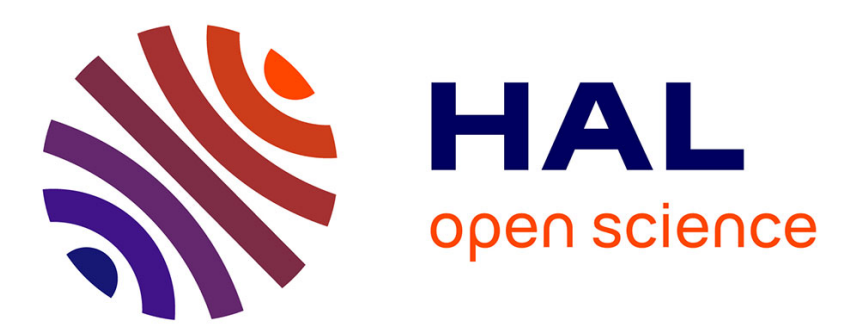

\title{
Formulation du contact avec adhérence en élasticité non linéaire entre deux solides déformables
}

\author{
A. S. Caro-Bretelle, M. Cocu, Yann Monerie
}

\section{To cite this version:}

A. S. Caro-Bretelle, M. Cocu, Yann Monerie. Formulation du contact avec adhérence en élasticité non linéaire entre deux solides déformables. Comptes Rendus de l'Académie des Sciences - Series IIB - Mechanics-Physics-Astronomy, 2000, 328 (3), pp.203-208. 10.1016/S1287-4620(00)00124-1 . hal03143957

\section{HAL Id: hal-03143957 \\ https://hal.science/hal-03143957}

Submitted on 28 May 2021

HAL is a multi-disciplinary open access archive for the deposit and dissemination of scientific research documents, whether they are published or not. The documents may come from teaching and research institutions in France or abroad, or from public or private research centers.
L'archive ouverte pluridisciplinaire HAL, est destinée au dépôt et à la diffusion de documents scientifiques de niveau recherche, publiés ou non, émanant des établissements d'enseignement et de recherche français ou étrangers, des laboratoires publics ou privés. 


\title{
Formulation du contact avec adhérence en élasticité non linéaire entre deux solides déformables
}

\author{
A.S. BRETELLE ${ }^{a}$, M. COCU ${ }^{b}$, Y. MONERIE ${ }^{a}$ \\ ${ }^{a}$ Laboratoire de mécanique et d'acoustique CNRS 31, chemin Joseph-Aiguier, 13402 Marseille cedex 20, \\ France \\ Courriel : bretelle@Ima.cnrs-mrs.fr; monerie@Ima.cnrs-mrs.fr \\ ${ }^{b}$ LMA-CNRS et université de Provence, Marseille, France \\ Courriel : cocu@Ima.cnrs-mrs.fr
}

Résumé. Dans le cadre de la mécanique non linéaire, grâce à la cinématique du contact introduite par A. Curnier, Q.C. He et J.J. Téléga, nous proposons une formulation thermodynamique eulérienne, conduisant à un problème aux limites couplant le contact unilatéral, le frottement et l'adhérence entre deux solides hyperélastiques, l'adhérence étant décrite par une variable d'état interne introduite par M. Frémond. Dans le cas particulier du contact sans frottement entre un solide hyperélastique et un support rigide plan on établit un résultat d'existence pour le problème de minimum associé, quand l'évolution de l'intensité d'adhérence est donnée par une loi «statique».

contact unilatéral / frottement / adhérence / grandes déformations

\section{Formulation of contact between two continuous bodies with adhesion in finite deformations}

\begin{abstract}
Within the framework of finite deformations and using an approach to the kinematics of contact due to A. Curnier, Q.C. He and J.J. Téléga, we propose a spatial thermodynamic formulation for the problem coupling unilateral condition, friction and adhesion. The adhesion is characterized by its intensity introduced by M. Frémond. In the case of frictionless contact between an hyperelastic body and a plane rigid support, with a particular 'static' law for the evolution of the intensity of adhesion, the problem can be reduced to a minimization one for which we can show the existence of a solution.
\end{abstract}

unilateral contact / friction / adhesion / finite deformations

\section{Abridged English version}

We propose a thermodynamic formulation of a quasistatic problem coupling unilateral contact, friction and adhesion between two hyperelastic bodies within the framework of finite deformations. According to M. Frémond [4] the adhesion is characterized by its intensity $\beta^{\varphi}$ which may vary from 1 (perfect adhesion) to 0 (no adhesion).

The present work extends the results of M. Raous et al. [6,7] which were established for a model of unilateral contact with friction and adhesion between two deformable bodies under the assumption of small deformations. 
In the framework of finite deformations we define the contact vector distance $\mathrm{d}^{\varphi}$, following A. Curnier et al. [1], Q.C. He et al. [2], A. Klarbring [3], by (2) via the orthogonal projection of a point belonging to one of the candidate potential contact surfaces onto the other one. Using the above construction, we are able to construct a frame, depending on the deformations of both bodies, with respect to which the quantities will be decomposed. This local approach is suitable for defining the condition of impenetrability via a signed contact distance vector. Another approach, proposed by P.G. Ciarlet and J. Nečas [5], consists in adopting a global formulation by considering an unilateral contact condition under the constraint to stay within a set. This does not seem to be helpful for a numerical investigation, so that we prefer to follow this local approach $[1,2]$. Using the distance $\mathrm{d}^{\varphi}$ we define an objective relative velocity by (3) and as we are able to deduce the contact behaviour for one surface with respect to the other one from the generalized action-reaction principle [1], it is sufficient to characterize the contact on one of the surfaces.

After employing the two principles of thermodynamics in any isothermal evolution of the system and choosing the Hencky's tensor as strain tensor to study the hyperelastic bodies, we obtain the ClausiusDuhem inequalities (7) for two solids and for one surface. With a particular choice of the surface density of free energy and according to the state laws (9) the unilateral contact condition (i.e. the penetration of two solids is excluded in compression situation) is obtained. The hyperelastic constitutive equation is deduced from the specific density of free energy which depends on the Hencky's tensor. We assume the pseudo potential of dissipation $\Phi^{\varphi^{1}}$ given by (10) to depend, among its variables, on the relative tangential velocity. By differentiating $\Phi^{\varphi^{1}}$ in the complementary laws we are conducted to the Coulomb's friction law with adhesion (17) and to the evolution law for the evolution of the intensity of adhesion (15). We only consider situations where the dissipative phenomena are due to both friction and adhesion.

The complete formulation of this spatial quasistatic problem is given by problem $\mathcal{P}^{E}$ (12) to (15). Finally, when one of the bodies is a plane rigid support, we propose a Lagrangian variational formulation, coupling unilateral contact condition, friction law and a differential inclusion for the intensity of adhesion. In the case of frictionless contact problems with a particular 'static' law for the intensity of adhesion, the corresponding variational problem can be reduced to the minimization problem (20) for which, using the approaches of J.M. Ball [8], P.G. Ciarlet and J. Nečas [5] and A. Curnier et al. [1], one can show an existence result.

\section{Introduction}

On considère un problème quasi statique couplant contact unilatéral, frottement de Coulomb et adhérence en grandes déformations entre deux solides hyperélastiques. Nous proposons, grâce à la cinématique du contact des grandes déformations introduite par A. Curnier et al. [1], Q.C. He et al. [2], A. Klarbring [3], une formulation thermodynamique eulérienne de ce problème nous conduisant à un problème aux limites couplant les équations d'équilibre, les conditions de contact unilatéral et de frottement de Coulomb et une inclusion differentielle caractérisant l'évolution de l'intensité d'adhérence [4]. Cette cinématique semble être la plus appropriée pour définir la condition de non interpénétration. L'approche alternative de P.G. Ciarlet et J. Nečas [5] suivant laquelle la condition de contact unilatéral est donnée par une restriction ensembliste semble difficilement exploitable numériquement.

Il nous a semblé nécessaire de donner cette formulation dans la configuration actuelle, configuration dans laquelle les lois de comportement surfacique trouvent une expression naturelle. Dans le cas particulier de l'hypothèse des petites perturbations on retrouve les résultats obtenus dans les travaux de $\mathrm{M}$. Raous et al. [6,7] pour un modèle couplant adhérence et frottement entre deux solides déformables.

Dans le cas du contact unilatéral sans frottement entre un solide hyperélastique et un support rigide plan et d'une loi «statique» pour l'intensité d'adhérence $\beta^{\varphi}$, une formulation par rapport à la configuration de référence du problème précédent nous permet d'étudier le problème aux limites par l'intermédiaire d'un problème de minimum. Cette démarche conduit à un problème classique de minimisation dans le cas de 
matériaux dissipatifs. On montre l'existence d'une solution de ce problème. Ce résultat étend ceux de J.M. Ball [8] et A. Curnier et al. [1] au contact adhésif.

\section{Formulations du modèle thermodynamique et du problème aux limites}

Dans ce qui suit, la variable $\alpha$ prend successivement les valeurs 1 et 2 . On considère deux solides déformables et $\Omega^{\alpha} \in \mathbb{R}^{3}$ leur configuration de référence de normales extérieures unitaires $\mathbf{n}^{\alpha}$. On note $\Omega^{\varphi^{\alpha}}$ les configurations actuelles des solides ayant subi la déformations $\varphi^{\alpha}$ et $\mathbf{n}^{\varphi^{\alpha}}$ leur normale extérieure unitaire. On décompose les frontières de $\Omega^{\alpha}$ et $\Omega^{\varphi^{\alpha}}$, supposées régulières, en des parties ouvertes disjointes notées respectivement $\Gamma^{\alpha}$ et $\Gamma^{\varphi^{\alpha}}=\varphi^{\alpha}\left(\Gamma^{\alpha}, t\right)$ telles que :

$$
\Gamma^{\alpha}=\bar{\Gamma}_{D}^{\alpha} \cup \bar{\Gamma}_{T}^{\alpha} \cup \bar{\Gamma}_{C}^{\alpha}, \quad \Gamma^{\varphi^{\alpha}}=\bar{\Gamma}_{D}^{\varphi^{\alpha}} \cup \bar{\Gamma}_{T}^{\varphi^{\alpha}} \cup \bar{\Gamma}_{C}^{\varphi^{\alpha}}
$$

Les corps sont soumis à des densités de forces volumiques et surfaciques : $\mathbf{f}^{\alpha}, \mathbf{T}^{\alpha}$ définies dans $\Omega^{\alpha}$ et respectivement sur $\Gamma_{T}^{\alpha}$ et $\mathbf{f}^{\varphi^{\alpha}}, \mathbf{T}^{\varphi^{\alpha}}$ données dans $\Omega^{\varphi^{\alpha}}$ et respectivement sur $\Gamma_{T}^{\varphi^{\alpha}}$. Les parties $\Gamma_{C}^{\alpha}$, $\Gamma_{C}^{\varphi^{\alpha}}=\varphi^{\alpha}\left(\Gamma_{C}^{\alpha}, t\right)$ sont les surfaces potentielles de contact des deux solides dans les deux configurations où les corps peuvent être en contact unilatéral. Sur $\Gamma_{D}^{\alpha}$ et $\Gamma_{D}^{\varphi^{\alpha}}$ on impose des déformations. On désigne $\operatorname{par} \mathbf{t}^{\alpha}, \mathbf{t}^{\varphi^{\alpha}}$ les densités de forces exercées le long des surfaces $\Gamma_{C}^{\alpha}, \Gamma_{C}^{\varphi^{\alpha}}$. On introduit une application $\varphi_{p}$ permettant de relier un point de $\Gamma^{\varphi^{1}}$, noté $\mathbf{x}^{\varphi^{1}}$, à son projeté sur $\Gamma_{C}^{\varphi^{2}}$ noté $\mathbf{x}^{\Pi^{\varphi}}$. On note $\mathbf{X}_{p}^{\varphi}$ l'application $\left(\varphi^{2}\right)^{-1} \circ \varphi_{p} \circ \varphi^{1}$ et $\mathbf{X}^{\Pi^{\varphi}}=\mathbf{X}_{p}^{\varphi}\left(\mathbf{X}^{1}\right)$ [1]. On considère une paramétrisation de la surface $\Gamma_{C}^{\varphi^{2}}$ et on définit $\boldsymbol{\xi}_{p}^{\varphi}$ comme étant le point correspondant à $\mathbf{X}^{\Pi^{\varphi}}$ dans l'espace de paramétrisation, ainsi qu'un repère $\left(\mathbf{n}^{\varphi}, \tau^{\varphi}\right)$ en ce point, où $\mathbf{n}^{\varphi}=\mathbf{n}^{\varphi^{2}}\left(\mathbf{x}^{\Pi^{\varphi}}\right)$ et $\boldsymbol{\tau}^{\varphi}=\left(\boldsymbol{\tau}_{1}^{\varphi}, \boldsymbol{\tau}_{2}^{\varphi}\right), \boldsymbol{\tau}_{\alpha}^{\varphi}$ sont des vecteurs linéairement indépendants et tangents à la surface $\varphi^{2}\left(\Gamma_{C}^{2}, t\right)$ au point considéré. On définit le vecteur distance de contact par :

$$
\mathbf{d}^{\varphi}\left(\mathbf{x}^{\varphi^{1}}, t\right)=\mathbf{x}^{\varphi^{1}}-\mathbf{x}^{\Pi^{\varphi}}\left(\mathbf{x}^{\varphi^{1}}, t\right)=\mathbf{d}^{\varphi}\left(\mathbf{X}^{1}, t\right)=\varphi^{1}\left(\mathbf{X}^{1}, t\right)-\varphi^{2}\left(\mathbf{X}^{\Pi^{\varphi}}\left(\mathbf{X}^{1}\right), t\right)
$$

que l'on peut décomposer en $\mathrm{d}^{\varphi}=d_{n^{\varphi}}^{\varphi} \mathbf{n}^{\varphi}$. On définit la vitesse relative généralisée suivante :

$$
\mathbf{V}_{\mathrm{re}}^{\varphi}=\dot{d}_{n \varphi}^{\varphi} \mathbf{n}^{\varphi}+\dot{\boldsymbol{\xi}}_{p_{k}}^{\varphi} \boldsymbol{\tau}_{k}^{\varphi}=\dot{d}_{n^{\varphi}}^{\varphi} \mathbf{n}^{\varphi}+\dot{\boldsymbol{\xi}}_{\boldsymbol{\tau} \varphi}^{\varphi}
$$

qui satisfait l'axiome d'indifférence matérielle. Cette définition de la distance privilégie un solide par rapport à l'autre et se place donc dans le contexte de l'approche 'maître/esclave'. On choisit ici les surfaces $\Gamma_{C}^{1}, \Gamma_{C}^{\varphi^{1}}$ comme surfaces maîtres et on déduit le comportement de $\varphi_{p}\left(\Gamma_{C}^{\varphi^{1}}\right)$ grâce à la loi d'action-réaction généralisée de A. Curnier et al. [1].

On considère le cas de matériaux hyperélastiques, homogènes (pour simplifier) et isotropes et l'on choisit d'exprimer la densité d'énergie libre $\psi^{\varphi^{\alpha}}$ en fonction du tenseur de Hencky $\mathbb{H}^{\varphi^{\alpha}}=L n \mathbb{V}^{\varphi}$ (où $\mathbb{V}^{\varphi}$ provient de la décomposition polaire de $\mathbb{F}^{\varphi}$ ) qui permet naturellement d'exprimer le tenseur contrainte de Cauchy comme la dérivée d'une énergie par rapport à cette variable [9]. Soit l'expression suivante de la dissipation intrinsèque volumique :

$$
d^{\varphi^{\alpha}}=-\rho^{\varphi^{\alpha}} \dot{\psi}^{\varphi^{\alpha}}+\sigma^{\varphi^{\alpha}}: \quad \dot{\mathbb{H}}^{\varphi^{\alpha}} \text { dans } \Omega^{\varphi^{\alpha}}
$$

où $\rho^{\varphi^{\alpha}}$ est la densité volumique des solides. Soit la densité surfacique d'énergie libre $\Psi^{\varphi^{1}}$ :

$$
\Psi^{\varphi^{1}}=\Psi^{\varphi^{1}}\left(d_{n^{\varphi}}^{\varphi}, \boldsymbol{\xi}_{\tau^{\varphi}}^{\varphi}, \beta^{\varphi}\right)=\frac{1}{\rho_{S}^{\varphi^{1}}}\left(I d_{K}\left(d_{n^{\varphi}}^{\varphi}\right)+I d_{P}\left(\beta^{\varphi}\right)\right)+\Psi_{0}^{\varphi^{1}}\left(d_{n^{\varphi}}^{\varphi}, \boldsymbol{\xi}_{\tau^{\varphi}}^{\varphi}, \beta^{\varphi}\right) \quad \operatorname{sur} \Gamma_{C}^{\varphi^{1}}
$$

où $K=\left\{d_{n} ; d_{n} \geqslant 0\right\}, P=\{\gamma ; 0 \leqslant \gamma \leqslant 1\}, I d_{K}, I d_{P}$ sont les fonctions indicatrices des ensembles $K, P$, et $\rho_{S}^{\varphi^{1}}$ est la densité surfacique du premier solide. Le terme $\Psi_{0}^{\varphi^{1}}$ est donné sur $\Gamma_{C}^{\varphi^{1}}$, convexe en $\left(d_{n^{\varphi}}^{\varphi}, \boldsymbol{\xi}_{\tau^{\varphi}}^{\varphi}\right)$ 
et en $\beta^{\varphi}$ et fini (ce sera par exemple une énergie élastique caractérisant une élasticité endommageable du contact, voir M. Raous et al. [6,7], sous l'hypothèse des petites déformations).

La dissipation intrinsèque surfacique s'exprime de la façon suivante :

$$
D^{\varphi^{1}}=-\rho_{S}^{\varphi^{1}} \dot{\Psi}^{\varphi^{1}}-t_{n^{\varphi}}^{\varphi^{1}} \dot{d}_{n^{\varphi}}^{\varphi}-\mathbf{t}_{\tau^{\varphi}}^{\varphi^{1}} \dot{\boldsymbol{\xi}}_{\tau^{\varphi}}^{\varphi} \quad \operatorname{sur} \Gamma_{C}^{\varphi^{1}}
$$

Les deux premiers principes de la thermodynamique conduisent aux inégalités de Clausius-Duhem pour toute évolution isotherme du système :

$$
\rho^{\varphi^{\alpha}} \dot{\psi}^{\varphi^{\alpha}}=\boldsymbol{\sigma}^{\varphi^{\alpha}}: \quad \dot{\mathbb{H}}^{\varphi^{\alpha}} \text { dans } \Omega^{\varphi^{\alpha}} \quad \text { et } \quad \rho_{S}^{\varphi^{1}} \dot{\Psi}^{\varphi^{1}} \leqslant-t_{n \varphi}^{\varphi^{1}} \dot{d}_{n \varphi}^{\varphi}-\mathbf{t}_{\boldsymbol{\tau} \varphi}^{\varphi^{1}} \cdot \dot{\boldsymbol{\xi}}_{\boldsymbol{\tau}^{\varphi}}^{\varphi} \quad \operatorname{sur} \Gamma_{C}^{\varphi^{1}}
$$

qui permettent de vérifier la positivité des dissipations intrinsèques.

En décomposant les forces thermodynamiques en parties réversibles et irréversibles on postule les lois d'état suivantes :

$$
\begin{aligned}
& \left(\boldsymbol{\sigma}^{\varphi^{\alpha}}\right)^{R}=\rho^{\varphi^{\alpha}} \frac{\partial \psi^{\varphi^{\alpha}}}{\partial \mathbb{H}^{\varphi^{\alpha}}} \quad \text { dans } \Omega^{\varphi^{\alpha}} \\
& -\left(t_{n^{\varphi}}^{\varphi^{1}}\right)^{R} \in \rho_{S}^{\varphi^{1}} \partial_{d_{n \varphi}^{\varphi}} \Psi^{\varphi^{1}}, \quad-\left(\mathbf{t}_{\boldsymbol{\tau}^{\varphi}}^{\varphi^{1}}\right)^{R} \in \rho_{S}^{\varphi^{1}} \partial_{\boldsymbol{\xi}_{\tau \varphi}^{\varphi}} \Psi^{\varphi^{1}}, \quad-G_{\beta} \in \rho_{S}^{\varphi^{1}} \partial_{\beta} \varphi \Psi^{\varphi^{1}} \quad \operatorname{sur} \Gamma_{C}^{\varphi^{1}}
\end{aligned}
$$

où $\partial_{y}$ désigne le sous-differentiel par rapport à la variable $y$. On choisit un potentiel de dissipation surfacique de la forme :

$$
\Phi^{\varphi^{1}}=\Phi^{\varphi^{1}}\left(\dot{\boldsymbol{\xi}}_{\boldsymbol{\tau}^{\varphi}}^{\varphi^{1}}, \dot{\beta}^{\varphi} ; \chi\right)=\frac{\mu}{\rho_{S}^{\varphi^{1}}}\left|t_{n^{\varphi}}^{\varphi^{1}}+\rho_{S}^{\varphi^{1}} \frac{\partial \Psi_{0}^{1}}{\partial d_{n^{\varphi}}^{\varphi}}\right|\left\|\dot{\boldsymbol{\xi}}_{\tau^{\varphi}}^{\dot{\varphi}}\right\|+\Phi_{0}^{\varphi^{1}}\left(\dot{\beta}^{\varphi} ; t_{n^{\varphi}}^{\varphi^{1}}, \beta^{\varphi}\right), \chi=\left(t_{n^{\varphi}}^{\varphi^{1}}, d_{n^{\varphi}}^{\varphi}, \boldsymbol{\xi}_{\tau^{\varphi}}^{\varphi}, \beta^{\varphi}\right)
$$

où $\Phi_{0}^{\varphi^{1}}$ est une fonction donnée objective, convexe et finie, son choix caractérisera le mode d'évolution de l'intensité d'adhérence. On peut ainsi formuler les lois complémentaires suivantes :

$$
\begin{aligned}
& \left(\boldsymbol{\sigma}^{\varphi^{\alpha}}\right)^{I R}=0 \quad \text { dans } \Omega^{\varphi^{\alpha}} \quad \text { et } \\
& \left(t_{n^{\varphi}}^{\varphi^{1}}\right)^{I R}=0, \quad-\left(\mathbf{t}_{\boldsymbol{\tau}^{\varphi}}^{\varphi^{1}}\right)^{I R} \in \rho_{S}^{\varphi^{1}} \partial_{\dot{\boldsymbol{\xi}}_{\tau \varphi}^{\varphi}} \Psi^{\varphi^{1}}, \quad G_{\beta} \in \rho_{S}^{\varphi^{1}} \partial_{\dot{\beta}^{\varphi}} \Phi^{\varphi^{1}} \quad \operatorname{sur} \Gamma_{C}^{\varphi^{1}}
\end{aligned}
$$

On déduit alors le problème quasi statique sous la forme générale suivante où l'on a noté par '*' la transformée de Legendre-Fenchel par rapport à la variable $\dot{\beta}^{\varphi}$ :

Problème $\mathcal{P}^{E}$. - Trouver $\varphi^{\alpha}, \beta^{\varphi}$ telles que $\varphi^{\alpha}(0)=\varphi_{0}^{\alpha}$ dans $\Omega^{\varphi^{\alpha}}$ et $\beta^{\varphi}(0)=\beta_{0}^{\varphi}$ sur $\Gamma_{C}^{\varphi^{1}}$ vérifiant les équations d'équilibre, la loi constitutive (8), les conditions aux limites sur $\Gamma_{D}^{\varphi^{\alpha}}$ et $\Gamma_{T}^{\varphi^{\alpha}}$ et les conditions suivantes pour tout $t \in] 0, T[$ et $\alpha=1,2$ :

$$
\begin{aligned}
& t_{n \varphi}^{\varphi^{1}}+\rho_{S}^{\varphi^{1}} \frac{\partial \Psi_{0}^{\varphi^{1}}}{\partial d_{n^{\varphi}}^{\varphi}} \geqslant 0, \quad d_{n \varphi}^{\varphi} \geqslant 0, \quad\left(t_{n^{\varphi}}^{\varphi^{1}}+\rho_{S}^{\varphi^{1}} \frac{\partial \Psi_{0}^{\varphi^{1}}}{\partial d_{n^{\varphi}}^{\varphi}}\right) d_{n^{\varphi}}^{\varphi}=0 \quad \operatorname{sur} \Gamma_{C}^{\varphi^{1}} \\
& \left\|\mathbf{t}_{\tau^{\varphi}}^{\varphi^{1}}+\rho_{S}^{\varphi^{1}} \frac{\partial \Psi_{0}^{\varphi^{1}}}{\partial \boldsymbol{\xi}_{\tau^{\varphi}}^{\varphi}}\right\| \leqslant \mu\left|t_{n^{\varphi}}^{\varphi^{1}}+\rho_{S}^{\varphi^{1}} \frac{\partial \Psi_{0}^{\varphi^{1}}}{\partial d_{n^{\varphi}}^{\varphi}}\right| \operatorname{sur} \Gamma_{C}^{\varphi^{1}} \\
& \text { et }\left\{\begin{array}{l}
\left\|\mathbf{t}_{\boldsymbol{\tau}^{\varphi}}^{\varphi^{1}}+\rho_{S}^{\varphi^{1}} \frac{\partial \Psi_{0}^{\varphi^{1}}}{\partial \boldsymbol{\xi}_{\tau^{\varphi}}^{\varphi}}\right\||<| t_{n^{\varphi}}^{\varphi^{1}}+\rho_{S}^{\varphi^{1}} \frac{\partial \Psi_{0}^{\varphi^{1}}}{\partial d_{n^{\varphi}}^{\varphi}} \mid \Rightarrow \dot{\boldsymbol{\xi}}_{\boldsymbol{\tau}^{\varphi}}^{\varphi}=\mathbf{0} \\
\left\|\mathbf{t}_{\boldsymbol{\tau}^{\varphi}}^{\varphi^{1}}+\rho_{S}^{\varphi^{1}} \frac{\partial \Psi_{0}^{\varphi^{1}}}{\partial \boldsymbol{\xi}_{\tau^{\varphi}}^{\varphi}}\right\|=\mu\left|t_{n^{\varphi}}^{\varphi^{1}}+\rho_{S}^{\varphi^{1}} \frac{\partial \Psi_{0}^{\varphi^{1}}}{\partial d_{n^{\varphi}}^{\varphi}}\right| \Rightarrow \exists \lambda \geqslant 0, \quad \dot{\boldsymbol{\xi}}_{\boldsymbol{\tau}^{\varphi}}^{\varphi}=\lambda\left(\mathbf{t}_{\boldsymbol{\tau}^{\varphi}}^{\varphi^{1}}+\rho_{S}^{\varphi^{1}} \frac{\partial \Psi_{0}^{\varphi^{1}}}{\partial \boldsymbol{\xi}_{\boldsymbol{\tau}^{\varphi}}^{\varphi}}\right)
\end{array}\right. \\
& \dot{\beta}^{\varphi} \in \partial_{G_{\beta}}\left(\rho_{S}^{\varphi^{1}} \Phi^{\varphi^{1}}\right)^{*}, \quad-G_{\beta} \in \rho_{S}^{\varphi^{1}} \partial_{\beta} \varphi \Psi^{\varphi^{1}} \quad \operatorname{sur} \Gamma_{C}^{\varphi^{1}}
\end{aligned}
$$




\section{Résultat d'existence dans un cas particulier : contact sans frottement avec un obstacle plan}

Dans le cas du contact entre un solide hyperélastique et un support rigide plan en contact à l'instant initial, les variables d'état du système écrit par rapport à la configuration de référence, se réduisent aux variables $\boldsymbol{\xi}_{\tau^{\varphi}}^{\varphi}=\mathbf{u}_{\tau}^{\varphi}+\mathbf{c}$, $\mathbf{c}$ constante, $d_{n^{\varphi}}^{\varphi}=u_{n}^{\varphi}, \beta=\beta^{\varphi} \circ \varphi^{1}$ où $\mathbf{u}^{\varphi}(\mathbf{X}, t)=\varphi(\mathbf{X}, t)-\mathbf{X}$ et $u_{n}^{\varphi}(0)=0$. Dans ce cas, $\Psi_{0}=\widehat{\Psi}_{0}\left(\mathbf{u}_{\tau}^{\varphi}, u_{n}^{\varphi}, \beta\right)$ et pour simplifier l'expression, on a éliminé les indices correspondant à $\alpha=1$ ( $\alpha=2$ étant l'indice du plan). En utilisant le tenseur non symétrique de Piola-Kirchhoff et la densité d'énergie $\widehat{\mathbf{W}}=\rho \psi$, le problème $\mathcal{P}^{E}$ est ramené au problème suivant :

Problème $\mathcal{P}^{L}$. - Trouver $\varphi, \beta$ telles que $\varphi(0)=\varphi_{0}$ dans $\Omega, \beta(0)=\beta_{0}$ sur $\Gamma_{C}$ et vérifiant les équations d'équilibre, la loi constitutive, les conditions aux limites sur $\Gamma_{D}$ et $\Gamma_{T}$ et les conditions suivantes pour tout $t \in] 0, T[:$

$$
\begin{gathered}
t_{n}+\rho_{S} \frac{\partial \widehat{\Psi}_{0}}{\partial u_{n}^{\varphi}} \geqslant 0, \quad u_{n}^{\varphi} \geqslant 0, \quad\left(t_{n}+\rho_{S} \frac{\partial \widehat{\Psi}_{0}}{\partial u_{n}^{\varphi}}\right) u_{n}^{\varphi}=0 \quad \operatorname{sur} \Gamma_{C} \\
\left\|\mathbf{t}_{\boldsymbol{\tau}}+\rho_{S} \frac{\partial \widehat{\Psi}_{0}}{\partial \mathbf{u}_{\tau}^{\varphi}}\right\| \leqslant \mu\left|t_{n}+\rho_{S} \frac{\partial \widehat{\Psi}_{0}}{\partial u_{n}^{\varphi}}\right| \quad \operatorname{sur} \Gamma_{C} \\
\text { et }\left\{\begin{array}{c}
\left\|\mathbf{t}_{\boldsymbol{\tau}}+\rho_{S} \frac{\partial \widehat{\Psi}_{0}}{\partial \mathbf{u}_{\tau}^{\varphi}}\right\|<\mu\left|t_{n}+\rho_{S} \frac{\partial \widehat{\Psi}_{0}}{\partial u_{n}^{\varphi}}\right| \Rightarrow \dot{\mathbf{u}}_{\tau}^{\varphi}=\mathbf{0} \\
\left\|\mathbf{t}_{\boldsymbol{\tau}}+\rho_{S} \frac{\partial \widehat{\Psi}_{0}}{\partial \mathbf{u}_{\tau}^{\varphi}}\right\|=\mu\left|t_{n}+\rho_{S} \frac{\partial \widehat{\Psi}_{0}}{\partial u_{n}^{\varphi}}\right| \Rightarrow \exists \lambda \geqslant 0, \quad \dot{\mathbf{u}}_{\tau}^{\varphi}=\lambda\left(\mathbf{t}_{\boldsymbol{\tau}}+\rho_{S} \frac{\partial \widehat{\Psi}_{0}}{\partial \mathbf{u}_{\tau}^{\varphi}}\right) \\
\dot{\beta} \in \partial_{G_{\beta}}\left(\rho_{S} \widehat{\Phi}\right)^{*}, \quad-G_{\beta} \in \rho_{S} \partial_{\beta} \widehat{\Psi} \quad \operatorname{sur} \Gamma_{C}
\end{array}\right.
\end{gathered}
$$

En considérant le problème sans frottement, avec une loi «statique» où $\beta$ est une fonction seulement de $\varphi$, on retrouve alors le problème de minimisation classiquement étudié :

$$
\inf _{\psi \in \mathcal{Q}}\{I(\psi)+J(\psi)-L(\psi)\}
$$

où :

$$
\begin{aligned}
\mathcal{Q}=\{\psi & \in W^{1, p}\left(\Omega, \mathbb{R}^{3}\right) ; \operatorname{Cof} \nabla \psi \in L^{q}\left(\Omega, \mathbb{R}^{9}\right), \operatorname{det} \nabla \psi \in L^{r}(\Omega), \operatorname{det} \nabla \psi>0 \text { p.p. dans } \Omega, \\
& \left.=\varphi_{D} \text { p.p. sur } \Gamma_{D}, u_{n}^{\psi} \geqslant 0 \text { p.p. sur } \Gamma_{C}\right\}
\end{aligned}
$$

et $p, q, r$ convenablement choisis :

$$
I(\psi)=\int_{\Omega} \widehat{\mathbf{W}}(\nabla \psi(\mathbf{X})) \mathrm{d} \mathbf{X}, \quad J(\psi)=\int_{\Gamma_{C}} \rho_{S} \widehat{\Psi}_{0}\left(\mathbf{u}^{\psi}\right) \mathrm{d} A, \quad L(\psi)=\int_{\Omega} \mathbf{f} \cdot \psi \mathrm{d} \mathbf{X}+\int_{\Gamma_{T}} \mathbf{T} \cdot \psi \mathrm{d} A
$$

Sous des hypothèses de faible semi-continuité inférieure pour $J$ et de polyconvexité sur $\widehat{\mathbf{W}}$ et si mes $\left(\Gamma_{D}\right)>0$, on établit, un résultat d'existence pour ce problème.

\section{Références bibliographiques}

[1] Curnier A., He Q.C., Téléga J.J., Formulation of unilateral contact between two elastic bodies undergoing finite deformations, C. R. Acad. Sci. Paris Série II 314 (1992) 1-6.

[2] He Q.C., Téléga J.J., Curnier A., Unilateral contact of two solids subject to large deformations: formulation and existence results, Proc. R. Soc. London 452 A (1996) 2691-2727.

[3] Klarbring A., Large displacement frictional contact: a continuum framework for finite element discretization, Eur. J. Mech. A Solids 15 (1995) 237-253. 
[4] Frémond M., Adhérence des solides, J. Méc. Théor. Appl. 6 (1987) 383-407.

[5] Ciarlet P.G., Nečas J., Unilateral problems in nonlinear three-dimensional elasticity, Arch. Rat. Mech. Anal. 87 (1985) 319-338.

[6] Raous M., Cangemi L., Cocu M., Un modèle couplant adhérence et frottement pour le contact entre deux solides déformables, C. R. Acad. Sci. Paris Série IIb 325 (1997) 503-509.

[7] Raous M., Cangemi L., Cocu M., A consistent model coupling adhesion, friction, and unilateral contact, Comp. Meth. Appl. Mech. Engrg. 177 (1999) 383-399.

[8] Ball J.M., Convexity conditions and existence theorems in nonlinear elasticity, Arch. Rat. Mech. Anal. 63 (1977) $337-403$.

[9] Bretelle A.S., Formulation et résolution des problèmes de contact avec frottement et adhérence en grandes déformations, Thèse de doctorat de l'université d'Aix-Marseille II, 1999. 